\title{
Macroalgas de riachos da Serra da Prata, leste do Estado do Paraná, Sul do Brasil
}

\author{
Cleto Kaveski Peres ${ }^{1}$, Ciro Cesar Zanini Branco ${ }^{1,2}$ e Rogério Antonio Krupek ${ }^{1}$
}

Recebido em 7/11/2006. Aceito em 11/06/2007

\begin{abstract}
RESUMO - (Macroalgas de riachos da Serra da Prata, leste do Estado do Paraná, Sul do Brasil). Estudos florísticos das comunidades de macroalgas lóticas no Brasil são quase que exclusivamente baseadas em material do Estado de São Paulo. Informações sobre macroalgas de riachos são escassos no Estado do Paraná. Neste contexto, o presente trabalho teve como objetivo realizar o levantamento florístico das comunidades de macroalgas de riachos da Serra da Prata, uma área bem preservada e protegida de Floresta Ombrófila Densa. As amostras foram realizadas em 14 segmentos de riachos. O levantamento resultou na identificação de 19 táxons (15 infragenéricos, três genéricos e uma fase do ciclo de vida de Batrachospermum spp.), distribuídos em quatro divisões. Cyanophyta foi a divisão com maior número de representantes (58\% das espécies) e Microcoleus subtorulosus Gomont ex Gomont foi a espécie mais bem distribuída. A maioria dos táxons encontrados (53\%) são primeiros registros no Estado do Paraná, ao passo que alguns outros são considerados de ocorrência rara em riachos brasileiros. Estes resultados reforçam a importância e a necessidade de mais estudos florísticos e taxonômicos para que se amplie o conhecimento sobre a biodiversidade das comunidades de macroalgas de riacho no Brasil.
\end{abstract}

Palavras-chave: macroalgas, riachos, Cyanophyta, levantamento florístico

ABSTRACT - (Stream macroalgae from Serra da Prata, eastern Paraná State, Southern Brazil). Studies of stream macroalgal communities in Brazil are almost exclusively based on data from São Paulo state. There are no extensive studies concerning stream macroalgae in Paraná state. In this context, the main objective of this study was to survey the flora of stream macroalgal communities in the Serra da Prata, a well-preserved and protected area of dense Atlantic rain forest. Sampling was carried out in 14 stream segments. The survey resulted in the identification of 19 taxa (15 infrageneric, three generic and one life cycle stage of Batrachospermum spp.), distributed in four divisions. Cyanophyta was the division with the highest number of taxa (58\% of the species) and Microcoleus subtorulosus Gomont ex Gomont was the most widespread species. Most of the taxa identified (53\%) were recorded for the first time in Paraná state, while others were considered rare in Brazilian streams. These results reinforce the importance and necessity of more floristic and taxonomic studies in order to increase our knowledge of stream macroalgal community biodiversity.

Key words: macroalgae, streams, Cyanophyta, floristic survey

\section{Introdução}

As algas bentônicas desempenham um papel fundamental em ambientes lóticos (Giller \& Malmqvist 1998; Matthael et al. 2003; Brito et al. 2006), de forma que a porção macroscópica destes organismos tem despertado, nas últimas décadas, um grande interesse como objeto de estudo. Assim, diversos trabalhos vêm sendo desenvolvidos com a finalidade de conhecer de forma mais precisa a composição das comunidades de macroalgas lóticas (Sheath \& Burkholder 1985; Sheath et al. 1986; 1988; 1989; Sherwood \& Sheath 1999; Hu \& Xie 2006). No presente trabalho, o termo "macroalga" foi adotado a partir da definição proposta por Sheath \& Cole (1992).
No Brasil, vários trabalhos já foram realizados enfocando as macroalgas (e.g. Necchi Júnior et al. 1991; 1994; 2000; Branco \& Necchi Júnior 1996a; 1996b; 1998; Branco et al. 2001; 2005). Porém, estudos taxonômicos, com o objetivo de ampliar o conhecimento da biodiversidade destas comunidades algais presentes em ambientes lóticos são raros. Neste sentido, pode-se citar apenas as investigações realizadas por Necchi Júnior et al. (1997), onde foram estudadas as macroalgas da região noroeste do Estado de São Paulo, e Branco \& Necchi Júnior (1996a), que estudaram as macroalgas da porção oriental da Mata Atlântica do mesmo Estado. Em adição, alguns estudos que trataram de grupos taxonômicos específicos, que abrigam representantes considerados como sendo macroalgas

\footnotetext{
1 Universidade Estadual Paulista "Júlio de Mesquita Filho", Faculdade de Ciências e Letras, Departamento de Ciências Biológicas, Av. Dom Antônio 2100, 19806-900 Assis, SP, Brasil

2 Autor para correspondência: czbranco@assis.unesp.br
} 
típicas, podem ser apontados como sendo importantes contribuições para o conhecimento da diversidade apresentada pelas comunidades de tais organismos. Entre eles destacam-se: Azevedo et al. (1996) e Branco et al. (1999) estudando diversos grupos dentro das Cyanophyceae; Necchi Júnior (1989) e Necchi Júnior \& Bicudo (1992) investigando Rhodophyta de águas continentais; Dias (1986; 1987; 1990; 1991; 1992) sobre as Chlorophyta filamentosas; Sant'anna (1988) estudando Scytonemataceae e Branco et al. (2002) sobre as Chaetophoraceae.

Considerando a carência de estudos com macroalgas no sul do Brasil, somada à necessidade de estudos taxonômicos deste importante grupo de produtores primários em águas correntes, o presente trabalho foi proposto com o objetivo de conhecer a biodiversidade das comunidades de macroalgas dos ambientes lóticos de uma área de Floresta Ombrófila Densa da região leste do Estado do Paraná.

\section{Material e métodos}

O estudo sobre as comunidades de macroalgas, a que se refere este trabalho, realizou-se na a Serra da Prata, leste do Estado do Paraná, Brasil. A área de estudo é quase totalmente representada pelo Parque Nacional Saint-Hilaire/Lange, o qual possui uma área de aproximadamente 25.000 ha, estando localizado entre os municípios de Matinhos, Guaratuba, Morretes e Paranaguá (IBAMA 2003). A vegetação que compõe a Serra da Prata é composta basicamente pela Floresta Ombrófila Densa (IBGE 1992).

O clima na porção leste do Estado do Paraná é influenciado diretamente pelas massas de ar quente e úmido do Oceano Atlântico, pertencendo à classificação Af (t) de Köeppen (Roderjan et al. 2002). Nesta região não há estação seca e as precipitações médias anuais podem exceder $3.000 \mathrm{~mm}$, apresentando as maiores precipitações do Estado (Pró-Atlântica 2002).

$\mathrm{Na}$ área total da Serra da Prata foram analisados 14 pontos de amostragem e, destes, oito foram visitados em duas ocasiões (Tab. 1). Tais amostragens foram realizadas com base na técnica da transeção (Necchi Júnior et al. 1995; Necchi Júnior et al. 2003) e, além da análise das comunidades de macroalgas presas aos substratos formadores do leito dos riachos, as seguintes variáveis ambientais foram medidas diretamente em campo: temperatura, turbidez, condutividade específica, $\mathrm{pH}$, oxigênio dissolvido, com o auxílio do analisador de qualidade da água com sonda multiparâmetro, Horiba U-10; velocidade da correnteza usando um fluxômetro mecânico, General Oceanics, 2030R; profundidade, com o auxílio de uma régua centimétrica. Ainda, foram anotados o tipo de substrato utilizado como suporte para a fixação das macroalgas (segundo as classes de partículas dadas por Gordon et al. 1992) e o sombreamento marginal (segundo as categorias propostas por DeNicola et al. 1992). Os outros seis pontos de amostragem foram visitados uma única vez, onde foram conduzidas amostragens exclusivamente qualitativas das comunidades de macroalgas, sem o registro de informações sobre dados ambientais.

Os espécimes encontrados ao longo das amostragens foram coletados e preservados em formaldeído a 4\%. As observações microscópicas foram realizadas com auxílio de microscópio trinocular LEICA, modelo DM1000. Para as análises morfométricas e ilustrações (fotomicrografias), foi utilizado um sistema de câmera de vídeo digital LEICA, modelo DFC-280, acoplado a um microcomputador com o software LEICA IM-50, específico para análise de imagem. A classificação das espécies em divisões seguiu o sistema de Hoek et al. (1995), exceto para Cyanophyta (Komárek \& Anagnostidis 1986; 1989 e Anagnostidis \& Komárek 1988; 1990). As amostras foram herborizadas em meio líquido (formaldeído 4\%) e depositadas no Herbário da Universidade Federal do Paraná (UPCB).

Para cada táxon identificado são apresentadas as seguintes informações: a) breve descrição; b) o número de tombamento do material examinado, c) comentários taxonômicos (quando necessário); d) pontos de amostragem onde ocorreram na Serra da Prata; e) características físicas e químicas dos riachos, exceto para os táxons coletados apenas nos riachos onde foram conduzidas amostragens qualitativas.

\section{Resultados}

\section{CYANOPHYTA}

Blennothrix komarekii Branco et Montejano, Algolog. Stud. 121. 2006.

Fig. 1

Talo formado por filamentos densamente emaranhados, longos, curvados, pseudo-ramificados, coloração verde ou azul brilhante. Bainha fina e incolor no ápice, espessa e de cor castanha na base. Tricomas 6,3-10,2 um diâm., levemente constritos nos septos, levemente atenuados para o ápice. Células mais curtas que largas, 2,1-4,5 um compr., compr./diâm. 0,3-0,7. 
Tabela 1. Localização dos pontos de amostragem na Serra da Prata, PR, Brasil.

\begin{tabular}{|c|c|c|}
\hline Pontos de amostragem & Localização & Datas das amostragens \\
\hline 1 & $\begin{array}{l}\text { Município de Matinhos, Cachoeira do Tigre - Rio Cachoeira } \\
25^{\circ} 44^{\prime} 27^{\prime} \mathrm{S}, 48^{\circ} 36^{\prime} 66^{\prime \prime} \mathrm{W}\end{array}$ & 2.vii.2005; 22.ii.2006 \\
\hline 2 & $\begin{array}{l}\text { Município de Matinhos, riacho atrás do Pesque-pague das Tilápias } \\
25^{\circ} 44^{\prime} 80^{\prime \prime} \mathrm{S}, 48^{\circ} 35^{\prime} 92^{\prime} \mathrm{W}\end{array}$ & 2.vii.2005; 22.ii.2006 \\
\hline 3 & $\begin{array}{l}\text { Município de Matinhos, Rio Cambará } \\
\text { 254'53's, } 48^{\circ} 35^{\prime} 86^{\prime} \text { W }\end{array}$ & 2.vii.2005; 22.ii.2006 \\
\hline 4 & $\begin{array}{l}\text { Município de Paranaguá, Rio da Colônia Pereira } \\
25^{\circ} 41^{\prime} 51^{\prime \prime} \mathrm{S}, 48^{\circ} 35^{\prime} 14^{\prime} \text { W }\end{array}$ & 2.vii.2005; 22.ii.2006 \\
\hline 5 & $\begin{array}{l}\text { Município de Paranaguá, Rio das Pombas } \\
25^{\circ} 39^{\prime} 18^{\prime \prime} \mathrm{S}, 48^{\circ} 35^{\prime} 42^{\prime} \text { W }\end{array}$ & 3.vii.2005; 23.ii.2006 \\
\hline 6 & $\begin{array}{l}\text { Município de Paranaguá, Rio da Colônia Maria Luisa } \\
25^{\circ} 38^{\prime} 63^{\prime} \text { S, } 48^{\circ} 36^{\prime} 05^{\prime \prime} \mathrm{W}\end{array}$ & 3.vii.2005; 23.ii.2006 \\
\hline 7 & $\begin{array}{l}\text { Município de Paranaguá, riacho na estrada de acesso ao Morro Inglês } \\
25^{\circ} 36^{\prime} 24^{\prime \prime} S, 48^{\circ} 37^{\prime} 66^{\prime} \mathrm{W}\end{array}$ & 3.vii.2005; 23.ii.2006 \\
\hline 8 & $\begin{array}{l}\text { Município de Matinhos, Rio Indaial } \\
25^{\circ} 47^{\prime} 79^{\prime \prime} \mathrm{S}, 48^{\circ} 34^{\prime} 01^{\prime \prime} \mathrm{W}\end{array}$ & 3.vii.2005; 23.ii.2006 \\
\hline 9 & $\begin{array}{l}\text { Município de Guaratuba, afluente do Rio do Henrique } \\
25^{\circ} 40^{\prime} 54^{\prime \prime} \mathrm{S}, 48^{\circ} 42^{\prime} 15^{\prime \prime} \mathrm{W}\end{array}$ & 26.xi.2005 \\
\hline 10 & $\begin{array}{l}\text { Município de Guaratuba, Rio do Henrique - próximo à foz } \\
25^{\circ} 40^{\prime} 55^{\prime} \mathrm{S}, 48^{\circ} 41^{\prime} 52^{\prime \prime} \mathrm{W}\end{array}$ & 26.xi.2005 \\
\hline 11 & $\begin{array}{l}\text { Município de Guaratuba, afluente do Rio do Henrique } \\
25^{\circ} 40^{\prime} 40^{\prime \prime S}, 48^{\circ} 41^{\prime} 51^{\prime \prime} \mathrm{W}\end{array}$ & 26.xi.2005 \\
\hline 12 & $\begin{array}{l}\text { Município de Guaratuba, afluente do Rio do Henrique } \\
25^{\circ} 40^{\prime} 39^{\prime \prime} \mathrm{S}, 48^{\circ} 41^{\prime} 10^{\prime \prime} \mathrm{W}\end{array}$ & 26.xi.2005 \\
\hline 13 & $\begin{array}{l}\text { Município de Guaratuba, Rio do Henrique } \\
25^{\circ} 40^{\prime} 37^{\prime} \text { S, } 48^{\circ} 41^{\prime} 03^{\prime \prime} \mathrm{W}\end{array}$ & 26.xi.2005 \\
\hline 14 & $\begin{array}{l}\text { Município de Guaratuba, Rio Cubatãozinho } \\
25^{\circ} 40^{\prime} 25^{\prime} \mathrm{S}, 48^{\circ} 43^{\prime} 42^{\prime} \mathrm{W}\end{array}$ & 26.xi.2005 \\
\hline
\end{tabular}

Conteúdo celular azul brilhante ou azul pálido, finamente granulado. Célula apical cônica ou arredondada, sem caliptra.

Material examinado: UPCB 53123, 53127, 53129.

Comentários: esta espécie foi recentemente descrita por Branco \& Montejano (2006) através de materiais do Estado de São Paulo e do México, sendo este, portanto, o terceiro registro histórico da espécie.

Distribuição na Serra da Prata: Pontos de amostragem 4, 6 e 7.

Características dos riachos $(\mathrm{N}=3)$ - temperatura da água $21,7-23,3{ }^{\circ} \mathrm{C}(\bar{x}=22,5)$; condutividade $25-28 \mu \mathrm{S} . c m^{-1}(\bar{x}=26)$; pH 6,6-6,8 ( $\left.\bar{x}=6,7\right)$; oxigênio dissolvido 5,7-6,9 mg.L $\mathrm{L}^{-1}(\bar{x}=6,4)$; turbidez 1-3 NTU $(\bar{x}=2,3)$; velocidade da correnteza $54-108 \mathrm{~cm} \cdot \mathrm{s}^{-1}$ ( $\bar{x}=78,9)$; profundidade $31,0-38,2 \mathrm{~cm}(\bar{x}=34,8)$; substratos rochosos; ambientes sombreados e fortemente sombreados.

Microcoleus subtorulosus Gomont ex Gomont, Ann. Sci. Nat. Bot. Sér. 7, 15: 360, 1892.

Fig. 2, 3
Talo formado por filamentos emaranhados, epifíticos sobre briófitas ou aderido a substrato rochoso, coloração azul esverdeada a azul escura. Filamentos (27,0-)50,0-132,0 $\mu$ m diâm. Bainha nem sempre visível, castanha ou hialina, espessa e com lamelação paralela. Tricomas paralelos ou torcidos, (2-)5-30 por bainha, constritos nos septos, 6,0-8,5 $\mu \mathrm{m}$ diâm. Células mais curtas que largas até quadráticas, (2,3-)5,0-9,0 $\mu \mathrm{m}$ compr., compr./diâm. 0,3-1,2. Célula apical cônicoarredondada ou cônico-aguda, (3,2-)6,5-13,0 $\mu \mathrm{m}$ compr. Material examinado: UPCB 53118, 53119, 53124, 53126, 53127, 53128, 53131, 53134, 53135.

Comentários: o material apresentou diâmetro do filamento maior do que o descrito $(15,0-50,0 \mu \mathrm{m})$ por Branco et al. (1999), entretanto, essa é uma característica muito variável dentro da espécie. O gênero Microcoleus Desmazières ex Gomont assemelha-se muito com Hydrocoleum Kützing ex Gomont, sendo que suas diferenças se restringem a uma fina estriação na bainha do último. Komárek \& Anagnostidis (2005), sugerem que estes gêneros devam 
ser revisados por métodos modernos (particularmente os que envolvam análises de características ultraestruturais e moleculares) para uma melhor separação.

Distribuição na Serra da Prata: Pontos de amostragem 2, 5, 6, 7, 8, 11 e 12.

Características dos riachos $(\mathrm{N}=7)$ - temperatura da água $19,8-23,2{ }^{\circ} \mathrm{C}(\bar{x}=21,3)$; condutividade $27-39 \mu \mathrm{S} . \mathrm{cm}^{-1}(\bar{x}=34) ; \mathrm{pH} 6,5-7,0(\bar{x}=6,7)$; oxigênio dissolvido 5,0-6,9 mg. $\mathrm{L}^{-1}(\bar{x}=5,7)$; turbidez 0-3 NTU $(\bar{x}=1,7)$; velocidade da correnteza $7-98 \mathrm{~cm} \cdot \mathrm{s}^{-1}$ $(\bar{x}=45,6)$; profundidade $11,8-56,7 \mathrm{~cm}(\bar{x}=25,1)$; substratos arenoso e rochoso; ambientes fortemente sombreados.

Nostoc verrucosum Vaucher ex Bornet et Flahault, Histoire dês Converves d'eau douce, 225, pl. 16, fig. 3, 1803.

Fig. 4, 5

Talo formado por colônia gelatinosa lobada, dura, aderida ao substrato, com superfície irregular, 1-5(-7) mm diâm., camada externa mais compacta que a interna. Filamentos dispostos de forma mais ou menos paralela ao centro da colônia, e densamente emaranhados na periferia. Bainha distinta na periferia, amarela ou hialina. Tricomas 3,0-5,0 $\mu$ m diâm. Células curtas, doliformes a subesféricas, 3,0-4,2 $\mu \mathrm{m}$ compr., compr./diâm. 0,7-1,3. Heterocitos esféricos, 5,0-8,5 $\mu \mathrm{m}$ compr., 5,0-8,2 $\mu$ m diâm., compr./diâm. 0,8-1,2.

Material examinado: UPCB 53116, 53117.

Distribuição na Serra da Prata: Ponto de amostragem 1.

Características dos riachos $(\mathrm{N}=2)$ - temperatura da água $20,0-22,2{ }^{\circ} \mathrm{C}(\bar{x}=21,1)$; condutividade 23-30 $\mu{\mathrm{S} . \mathrm{cm}^{-1}}^{-1}(\bar{x}=26)$; pH 5,7-6,9 ( $\left.\bar{x}=6,3\right)$; oxigênio dissolvido 5,9-6,5 mg. $\mathrm{L}^{-1}(\bar{x}=6,2)$; turbidez 0-1 NTU $(\bar{x}=0,5)$; velocidade da correnteza $60-230 \mathrm{~cm} \cdot \mathrm{s}^{-1}$ $(\bar{x}=144,8)$; profundidade $5,8-29,0 \mathrm{~cm}(\bar{x}=17,4)$; substratos rochosos; ambientes sombreados.

\section{Nostoc sp.}

Fig. 6, 7

Talo formado por colônia gelatinosa irregular, macia, com 1-2(-3) mm diâm. Filamentos longos, densamente emaranhados. Bainha indistinta, hialina. Tricomas 3,0-6,0 $\mu \mathrm{m}$ diâm. Células curtas, doliformes a subesféricas, 3,0-7,0 $\mu$ m compr., compr./diâm. 0,7-1,8. Heterocitos esféricos, 4,0-6,0 $\mu \mathrm{m}$ compr., 4,0-6,0 $\mu \mathrm{m}$ diâm., compr./diâm. 0,9-1,1. Acinetos não observados.

Material examinado: UPCB 53122.

Comentários: o material encontrado não apresentava acinetos o que impossibilitou a identificação em nível específico.

Distribuição na Serra da Prata: Ponto de amostragem 4.

Característica do riacho $(\mathrm{N}=1)$ - temperatura da água $20,3{ }^{\circ} \mathrm{C}$; condutividade $30 \mu \mathrm{S} . \mathrm{cm}^{-1}$; $\mathrm{pH} 6,9$; oxigênio dissolvido $5,8 \mathrm{mg} . \mathrm{L}^{-1}$; turbidez $0 \mathrm{NTU}$; velocidade da correnteza $84 \mathrm{~cm} \cdot \mathrm{s}^{-1}$; profundidade $84,0 \mathrm{~cm}$; substrato rochoso; ambiente sombreado.

Nostochopsis lobatus Wood ex Bornet et Flahault, Ann. Sci. Nat. Bot. Sér. 7, 5: 80, 1887.

Fig. 8

Talo formado por colônia gelatinosa vesiculosa, lobada, de coloração verde-azulada. Bainha hialina, homogênea. Tricomas flexuosos, constritos nos septos, 3,0-6,0 um diâm. Ramificações abundantes, flexuosas, freqüentemente mais largas na base do que no ápice. Células mais compridas que largas, 6,0-12 $\mu \mathrm{m}$ compr., compr./diâm. 1,2-2,4. Heterocitos arredondados ou cônicos, laterais ou intercalares, sempre pedicelados, 5,0-8,0 $\mu$ m diâm.

Material examinado: UPCB 53119.

Distribuição na Serra da Prata: Ponto de amostragem 2.

Características do riacho $(\mathrm{N}=1)$ - temperatura da água $22,4{ }^{\circ} \mathrm{C}$; condutividade $32 \mu \mathrm{S} . \mathrm{cm}^{-1} ; \mathrm{pH} 6,5$; oxigênio dissolvido $5,5 \mathrm{mg} . \mathrm{L}^{-1}$; turbidez $3 \mathrm{NTU}$; velocidade da correnteza $7 \mathrm{~cm} \cdot \mathrm{s}^{-1}$; profundidade $56,7 \mathrm{~cm}$; substrato arenoso; ambiente fortemente sombreado.

Phormidium aerugineo-caeruleum (Gomont) Anagnostidis et Komárek, Algolog. Stud. 50-53: 407, 1988.

Fig. 9

Talo formado por emaranhado de filamentos de coloração azul esverdeada a azul escura. Filamentos 2,8-6,1(-9,8) $\mu$ m diâm. Bainha hialina, fina, homogênea, às vezes ausente. Tricomas não constritos ou em raros casos fracamente constritos nos septos, 2,1-5,8(-7,0) $\mu \mathrm{m}$ diâm. Células mais curtas ou mais longas que largas, 1,2-4,2 $\mu \mathrm{m}$ compr., compr./diâm. (0,3-)0,5-1,3(-2,0). Conteúdo celular azul brilhante com pequenos e grandes grânulos azul esverdeados. Célula apical truncada ou arredondada, 1,9-5,0 $\mu \mathrm{m}$ compr.

Material examinado: UPCB 53121, 53123, 53127, 53128, 53129, 53131, 53136, 53141.

Distribuição na Serra da Prata: Pontos de amostragem 3, 4, 5, 6, 7, 8 e 13.

Características dos riachos $(\mathrm{N}=6)$ - temperatura da água $20,8-23,9{ }^{\circ} \mathrm{C}(\bar{x}=22,6)$; condutividade 
28-41 $\mu{\mathrm{S} . \mathrm{cm}^{-1}}^{-}(\overline{\mathrm{x}}=34)$; pH 6,6-7,0 ( $\left.\bar{x}=6,8\right)$; oxigênio dissolvido 5,2-6,9 mg. $\mathrm{L}^{-1}(\bar{x}=6,0)$; turbidez 0-3 NTU $(\bar{x}=2)$; velocidade da correnteza $19-108 \mathrm{~cm} \cdot \mathrm{s}^{-1}$ $(\bar{x}=76,0)$; profundidade $17,8-38,2 \mathrm{~cm}(\bar{x}=30,5)$; substratos rochosos; ambientes sombreados a fortemente sombreados.

Phormidium retzii (C. Agardh) Gomont ex Gomont, Ann. Sci. Nat. Bot., Sér. 7, 16: 175, 1892.

Fig. 10

Talo formado por emaranhado de filamentos de coloração azul esverdeada a azul escura. Filamentos 7,4-11,0 $\mu \mathrm{m}$ diâm. Bainha hialina, fina, às vezes ausente. Tricomas não constritos nos septos, 6,0-9,0 $\mu \mathrm{m}$ diâm. Células mais curtas que largas, 3,0-6,0 $\mu \mathrm{m}$ compr., compr./diâm. 0,4-0,8. Conteúdo celular azul com pequenos grânulos. Célula apical truncada ou arredondada, 3,1-8,0 $\mu \mathrm{m}$ compr.

Material examinado: UPCB 53116, 53141.

Comentários: Branco et al. (1999) comentam que esta espécie apresenta ampla variabilidade nas características morfométricas e que, por isso, existem várias incertezas sobre a precisão de sua circunscrição. Provavelmente, $P$. retzii represente um caso onde vários táxons estão reunidos sob uma única denominação.

Distribuição na Serra da Prata: Pontos de amostragem 1 e 5.

Características dos riachos $(\mathrm{N}=2)$ - temperatura da água $20,0-23,0{ }^{\circ} \mathrm{C}(\bar{x}=21,5)$; condutividade $30 \mu \mathrm{S} . \mathrm{cm}^{-1} \bar{x} ; \mathrm{pH} 6,3-6,9(\bar{x}=6,6)$; oxigênio dissolvido 6,0-6,5 mg.L $\mathrm{L}^{-1}(\bar{x}=6,2)$; turbidez 0-3 NTU ( $\left.\bar{x}=1,5\right)$; velocidade da correnteza $117-230 \mathrm{~cm} \cdot \mathrm{s}^{-1}(\bar{x}=173,5)$; profundidade $5,8-26,2 \mathrm{~cm}(\bar{x}=16,0)$; substratos rochosos; ambientes sombreado a fortemente sombreado.

Pleurocapsa fluviatilis Lagerheim, Notarisia 3: 430, 1888.

Fig. 11

Talo incrustante, firme, hemisférico, de coloração azul escura. Filamentos curtos, radiados ou paralelos. Células esféricas ou poligonais, 4,2-6,9 $\mu \mathrm{m}$ diâm., 2,7-4,8 $\mu \mathrm{m}$ compr., principalmente mais largas do que longas, compr./diâm. 0,6-0,8. Bainha incolor. Esporângios não observados.

Material examinado: UPCB 53127.

Distribuição na Serra da Prata: Ponto de amostragem 6.

Características do riacho $(\mathrm{N}=1)$ - temperatura da água $22,6{ }^{\circ} \mathrm{C}$; condutividade $27 \mu \mathrm{S} . \mathrm{cm}^{-1}$; pH 6,6; oxigênio dissolvido $6,9 \mathrm{mg} \cdot \mathrm{L}^{-1}$; turbidez $3 \mathrm{NTU}$; velocidade da correnteza $75 \mathrm{~cm} . \mathrm{s}^{-1}$; profundidade $31,0 \mathrm{~cm}$; substrato rochoso; ambiente fortemente sombreado.

Scytonema arcangeli Bornet et Flahault, Ann. Sci. Nat. Bot., Ser. 7, 5: 92, 1886.

Fig. 12

Talo formado por filamentos prostrados, de coloração verde azulada ou castanha. Filamentos com raras ramificações, 9,9-13,8 $\mu \mathrm{m}$ diâm. Falsas ramificações simples e duplas. Bainha hialina, espessa, às vezes com lamelação paralela e ondulada nas margens. Tricomas não constritos ou levemente constritos, 6,1-9,5 $\mu \mathrm{m}$ diâm. Células mais compridas que largas, 6,4-15,4 $\mu$ m compr., compr./diâm. 1,0-2,0. Conteúdo celular granulado, verde azulado. Heterocitos raros, quadrados ou cilíndricos, 8,7-16,3 $\mu \mathrm{m}$ compr., 6,0-10,0 $\mu \mathrm{m}$ diâm. Hormogônios presentes.

Material examinado: UPCB 53139.

Distribuição na Serra da Prata: Ponto de amostragem 1.

Características do riacho $(\mathrm{N}=1)$ - temperatura da água $22,2{ }^{\circ} \mathrm{C}$; condutividade $23 \mu \mathrm{S} . \mathrm{cm}^{-1}$; $\mathrm{pH} 5,7$; oxigênio dissolvido $5,9 \mathrm{mg} . \mathrm{L}^{-1}$; turbidez $1 \mathrm{NTU}$; velocidade da correnteza $59 \mathrm{~cm} \cdot \mathrm{s}^{-1}$; profundidade 29,0 cm; substrato rochoso; ambiente sombreado.

Symplocastrum friesii (C. Agardh) Forti, Syll. Myxoph.: 347. 1907.

Fig. 13

Talo formado por filamentos emaranhados, prostrados, de coloração verde a castanha. 1-5 tricomas paralelamente arranjados por bainha. Bainha espessa, hialina, afunilada e fechada no ápice, 10,6-22,3 Mm diâm. Tricomas constritos nos septos, 4,2-5,7 $\mu \mathrm{m}$ diâm. Células mais compridas que largas, 4,6-7,7 $\mu \mathrm{m}$ compr., compr./diâm. 1,0-1,6. Conteúdo celular verde brilhante, com grandes grânulos. Célula apical cônica ou cilíndrica com ápice arredondado, 7,2-10,2 $\mu$ m compr.

Material examinado: UPCB 53139.

Comentários: Branco et al. (1999), descreveram o mesmo material como Schizothrix friesii (C. Agardh) Gomont, porém, Komárek \& Anagnostidis (2005) transferiram esta e outras espécies do gênero Schizothrix para Symplocastrum. Neste mesmo trabalho, os autores comentam que os materiais brasileiros deveriam ser revisados.

Distribuição na Serra da Prata: Ponto de amostragem 1 . 

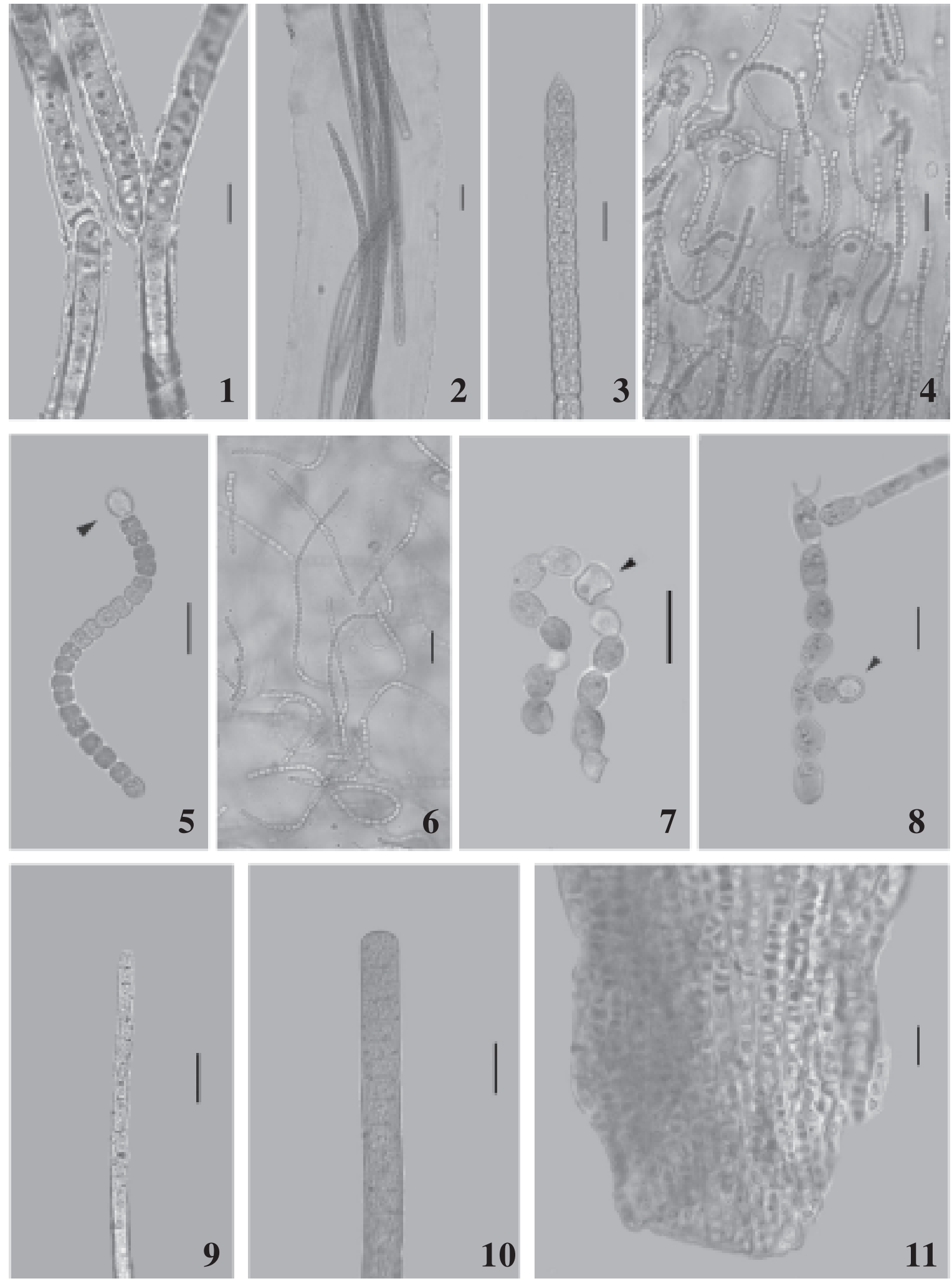

Figuras 1-11. 1. Blennothrix komarekii Branco et Montejano. 2-3. Microcoleus subtorulosus Gomont ex Gomont. 2. Filamento com vários tricomas. 3. Tricoma. 4-5. Nostoc verrucosum Vaucher ex Bornet et Flahault. 4. Aspecto dos tricomas no interior da colônia. 5. Tricoma mostrando heterocito basal (ponta de seta). 6-7. Nostoc sp. 6. Aspecto dos tricomas no interior da colônia. 7. Tricoma mostrando heterocito intercalar (ponta de seta). 8. Nostochopsis lobatus Wood ex Bornet et Flahault, parte do filamento mostrando heterocito pedunculado (ponta de seta). 9. Phormidium aerugineo-caeruleum (Gomont) Anagnostidis et Komárek. 10. Phormidium retzii (C. Agardh) Gomont ex Gomont. 11. Pleurocapsa fluviatilis Lagerheim. Barras = 2, 4 e $6=20$ m; 1, 3, 5, 7-11 = 10 $\mu$ m. 
Características do riacho $(\mathrm{N}=1)$ - temperatura da água $22,2{ }^{\circ} \mathrm{C}$; condutividade $23 \mu \mathrm{S} . \mathrm{cm}^{-1}$; $\mathrm{pH} 5,7$; oxigênio dissolvido $5,9 \mathrm{mg} . \mathrm{L}^{-1}$; turbidez $1 \mathrm{NTU}$; velocidade da correnteza $59 \mathrm{~cm} \cdot \mathrm{s}^{-1}$; profundidade 29,0 cm; substrato rochoso; ambiente sombreado.

Tolypothrix tenuis Kützing, Phyc. gene. 228. 1843. Fig. 14

Talo formado por uma massa de filamentos, 7,0-11,0 $\mu \mathrm{m}$ diâm. Falsas ramificações simples, longas, freqüentes e paralelas. Bainha com lamelação paralela, hialina. Tricomas fracamente constritos nos septos, 5,0-9,0 $\mu \mathrm{m}$ diâm. Células mais curtas que largas, 4,0-5,0 $\mu \mathrm{m}$ compr., compr./diâm. 0,6-1,0. Conteúdo celular azul brilhante, granulado. Heterocitos basais ou intercalares, cônicos ou arredondados, 7,0-11,0 $\mu \mathrm{m}$ compr., 5,0-11,0 $\mu$ m diâm., compr./diâm. 1.0-1,8.

Material examinado: UPCB 53136.

Comentários: o material examinado apresentou, como peculiaridade, as falsas ramificações sempre em posição paralela ao tricoma e não em posição oblíqua como é freqüentemente apresentado na literatura. Embora esta distinção tenha sido muito evidente, esse traço morfológico não é, aparentemente, relevante para invalidar a identificação específica.

Distribuição na Serra da Prata: Ponto de amostragem 13.

\section{CHLOROPHYTA}

Ecballocystis pulvinata Bohlin var. pulvinata, Sv. Vet.-Akad. Hand., 23(7): 7. 1897.

Fig. 15

Colônia formada por numerosos ramos eretos, dendróides, fortemente agregados. Base obcônica ou alargada. Células obcônicas, 9,0-14,8 $\mu \mathrm{m}$ diâm., 19,0-34,5 um compr., compr./diâm. 1,5-3,0. Cloroplastos 5-8, parietais, discóides. Somente duas células filhas formadas após a divisão celular. Paredes gelatinizadas das gerações mais antigas acumulam-se sob as células resultando, produzindo o arranjo estratificado típico.

Material examinado: UPCB 53116, 53120, 53121.

Distribuição na Serra da Prata: Pontos de amostragem 1 e 3 .

Características dos riachos $(\mathrm{N}=3)$ - temperatura da água $20,0-23,9{ }^{\circ} \mathrm{C}(\bar{x}=21,3)$; condutividade 27-30 $\mu{\mathrm{S} . \mathrm{cm}^{-1}}^{(}(\bar{x}=29) ; \mathrm{pH} 6,9-7,0(\bar{x}=7,0)$; oxigênio dissolvido 6,1-7,0 mg. $\mathrm{L}^{-1}(\bar{x}=6,5)$; turbidez 0-2 NTU $(\bar{x}=1)$; velocidade da correnteza $85-230 \mathrm{~cm} \cdot \mathrm{s}^{-1}$ $(\bar{x}=139,0)$; profundidade $5,8-34,0 \mathrm{~cm}(\bar{x}=23,9)$; substrato rochoso; ambientes sombreados.

\section{Spirogyra sp.}

Fig. 16

Filamentos formados por células cilíndricas. Células com 57,5-250,0 um compr., 17,1-42,5 ㅆm diâm., compr./diâm. 1,1-1,8. Cloroplastos em forma de hélice, parietais. Zigósporos não observados.

Material examinado: UPCB 53120, 53137.

Comentários: a grande amplitude no tamanho das células das duas populações examinadas nos permitiu especular que, possivelmente, devem se tratar de duas espécies distintas. Porém, neste gênero, os zigósporos (estruturas reprodutivas) são fundamentais para a identificação em nível específico, e os mesmos não foram encontrados nos espécimes coletados. Sendo assim, as identificações são apresentadas em nível de gênero, como um único grupo vegetativo.

Distribuição na Serra da Prata: Pontos de amostragem 3 e 14 .

Características do riacho $(\mathrm{N}=1)$ - temperatura da água $20,0{ }^{\circ} \mathrm{C}$; condutividade $30 \mu \mathrm{S} . \mathrm{cm}^{-1} ; \mathrm{pH} 7,0$; oxigênio dissolvido 7,0 mg.L $\mathrm{L}^{-1}$; turbidez $1 \mathrm{NTU}$; velocidade da correnteza $85 \mathrm{~cm} . \mathrm{s}^{-1}$; profundidade $31,8 \mathrm{~cm}$; substrato rochoso; ambiente sombreado.

\section{Zygnema sp.}

Fig. 17

Filamentos formados por células cilíndricas. Células com 18,6-87,5 um compr., 17,1-45,0 um diâm.; compr./diâm. 0,9-3,0. Cloroplastos, dois, estrelados, com um pirenóide cada.

Material examinado: UPCB 53138, 53139, 53121.

Comentários: neste gênero os zigósporos (estruturas reprodutivas) são fundamentais para a identificação em nível específico. No material examinado não foram encontrados espécimes férteis o que levou a tratá-lo em nível de gênero como um grupo vegetativo. Espécimes estéreis do gênero Zygogonium Kützing se assemelham muito com os de Zygnema C. Agardh, sendo que a característica distintiva mais evidente refere-se à forma dos cloroplastos, discóides nos primeiros e estrelados nos últimos. Porém, no material examinado, notam-se a presença de cloroplastos estrelados e discóides em células de um mesmo filamento. Este fato pressupõe que esta característica não é plenamente confiável. A identificação das populações encontradas, como Zygnema sp. se deve à predominância de cloroplastos do tipo estrelado. Entretando, sugerimos que estudos taxonômicos mais complexos sejam realizados com estes gêneros na busca de uma delimitação que permita 
uma melhor separação dos mesmos.

Distribuição na Serra da Prata: Pontos de amostragem 1 e 3.

Características do riacho $(\mathrm{N}=2)$ - temperatura da água $22,2-23,9{ }^{\circ} \mathrm{C}(\bar{x}=23,0)$; condutividade 23-27 $\mu \mathrm{S} . c m^{-1}(\bar{x}=25,0) ; \mathrm{pH} 5,7-7,0(\bar{x}=6,3)$; oxigênio dissolvido 5,9-6,1 mg. $\mathrm{L}^{-1}(\bar{x}=6,0)$; turbidez 1-2 NTU $(\bar{x}=1,5)$; velocidade da correnteza $59-102 \mathrm{~cm} . \mathrm{s}^{-1}$ $(\bar{x}=80,5)$; profundidade $29,0-34,0 \mathrm{~cm}(\bar{x}=31,5)$; substratos rochosos; ambientes sombreados.

Zygogonium ericetorum Kützing, Phyc. gene. 446. 1843.

Fig. 18

Filamentos formado por células cilíndricas. Células com 14,0-35,0 $\mu$ m compr., 14,0-22,0 $\mu$ m diâm., compr./ diâm. 0,6-1,8. Cloroplastos, dois, irregularmente arredondados, com um pirenóide central cada. Parede da célula em conjugação com espessamento formando peças em "H". Conjugação escalariforme. Zigósporos arredondados, 15,4-22,0 $\mu$ m diâm., com parede espessa, lisa e castanha.

Material examinado: UPCB 53133.

Distribuição na Serra da Prata: Ponto de amostragem 10.

\section{HETEROKONTOPHYTA}

Hydrosera whampöensis (Schwartz) Deby var. whampöensis, J. Microgr. 15: 209. 1891.

Fig. 19, 21

Filamentos densamente emaranhados, epífitos em briófitas. Valvas duplo-triangulares, ângulos arredondados com distintos campos de poros apicais. Superfície valvar ornamentada por aréolas irregulares. Valvas com $78,2-89,0 \mu \mathrm{m}$ por $62,2-70,4 \mu \mathrm{m}$. Células na vista pleural com 72,7-114,5 $\mu$ m compr., 69,5-85,1 $\mu$ m diâm.

Material examinado: UPCB 53122.

Distribuição na Serra da Prata: Ponto de amostragem 4.

Características do riacho $(\mathrm{N}=1)$ - temperatura da água $20,3{ }^{\circ} \mathrm{C}$; condutividade $30 \mu \mathrm{S} . \mathrm{cm}^{-1}$; pH 6,9; oxigênio dissolvido $5,8 \mathrm{mg} . \mathrm{L}^{-1}$; turbidez 0 NTU; velocidade da correnteza $84 \mathrm{~cm} \cdot \mathrm{s}^{-1}$; profundidade $41,5 \mathrm{~cm}$; substrato rochoso; ambiente sombreado.

Vaucheria geminata (Vaucher) De Candolle in

Lamarck et De Candolle, Fl. Franç., 2: 62. 1805.

Fig. 20

Talo formado por filamentos emaranhados, consistindo de sifões esparsamente ramificados,
77,5-112,5 um diâm. Gametóforo constituído de dois oogônios lateralmente dispostos a um anterídeo. Pedúnculo ereto, curvo na parte distal, 100,0-125,0 $\mu \mathrm{m}$ compr. Anterídeos circinados, tubulares, 60,0-85,0 $\mu \mathrm{m}$ compr., 30,0-35,0 $\mu \mathrm{m}$ diâm. Oogônios elipsóides a

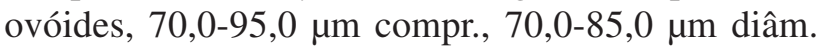
Oósporos não observados.

Material examinado: UPCB 53140, 53141.

Distribuição na Serra da Prata: Ponto de amostragem 5.

Características do riacho $(\mathrm{N}=2)$ - temperatura da água $19,8-23,0{ }^{\circ} \mathrm{C}(\bar{x}=21,4)$; condutividade 30-37 $\mu{\mathrm{S} . \mathrm{cm}^{-1}}^{-1}(\overline{\mathrm{x}}=33,5)$; pH 6,3-6,6 $(\bar{x}=6,5)$; oxigênio dissolvido 5,0-6,0 mg. $\mathrm{L}^{-1}(\bar{x}=5,5)$; turbidez 1-3 NTU $(\bar{x}=1,5)$; velocidade da correnteza $68-117 \mathrm{~cm} \cdot \mathrm{s}^{-1}$ $(\bar{x}=92,5)$; profundidade $15,6-26,2 \mathrm{~cm}(\bar{x}=20,9)$; substratos rochosos; ambientes fortemente sombreados.

\section{RHODOPHYTA}

Batrachospermum ambiguum Montagne, Ann. Sci. Nat. Bot. 14: 296. 1850.

Fig. 22, 23

Planta monóica, moderadamente mucilaginosa. Ramificação irregular, abundante. Verticilos doliformes, contíguos, 194-270 $\mu$ m diâm. Internó com 89-118 $\mu \mathrm{m}$ compr. Ramos primários retos, com 6-9 células. Células com 8,0-19,8 $\mu$ m compr., 3,8-5,8 $\mu$ m diâm. Ramos secundários numerosos, retos, inclinados sobre o interno. Espermatângios terminais, esféricos, 3,9-6,0 $\mu \mathrm{m}$ diâm. Ramo que sustenta o carpogônio torcido em hélice. Filamentos bracteóides numerosos. Tricogínio cilíndrico, pedicelado. Carposporófito central, menor que o ramo do verticilo. Carposporângios com 5,6-7,5 $\mu$ m diâm.

Material examinado: UPCB 53124.

Distribuição na Serra da Prata: Ponto de amostragem 5.

Características do riacho $(\mathrm{N}=1)$ - temperatura da água $19,8{ }^{\circ} \mathrm{C}$; condutividade $37 \mu \mathrm{S} . \mathrm{cm}^{-1}$; $\mathrm{pH}$ 6,6; oxigênio dissolvido 5,0 mg. $\mathrm{L}^{-1}$; turbidez 2 NTU; velocidade da correnteza $68 \mathrm{~cm} \cdot \mathrm{s}^{-1}$; profundidade $15,6 \mathrm{~cm}$; substrato rochoso; ambiente fortemente sombreado.

\section{Estágio 'Chantransia' de Batrachospermum spp.}

Fig. 24

Talo em forma de tufos de coloração azul avermelhada ou vermelha escura. Células com

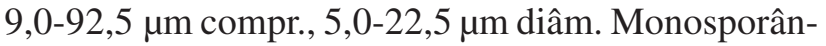
gios com 9,0-27,5 $\mu$ m diâm. 

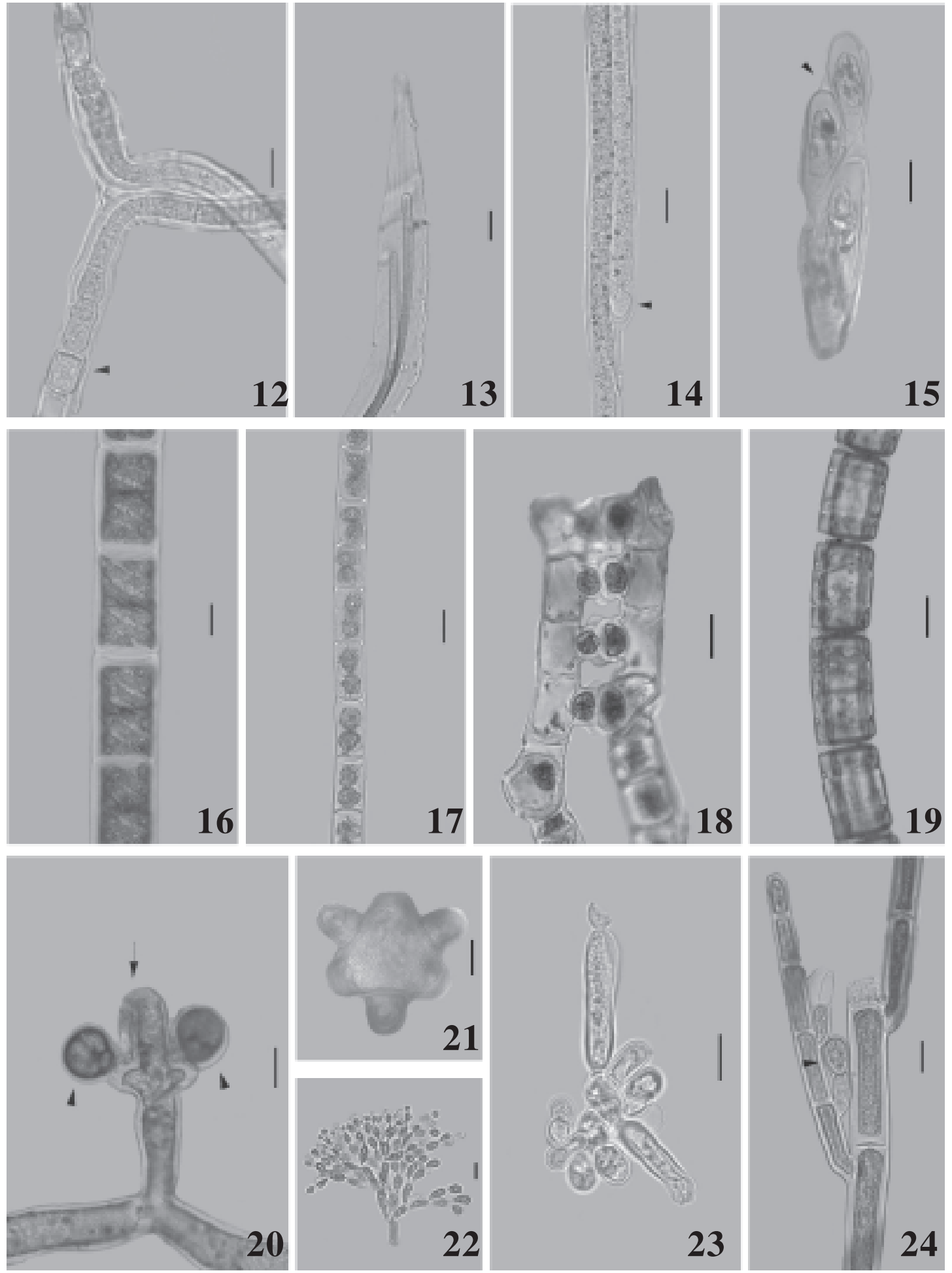

Figuras 12-24. 12. Scytonema arcangeli Bornet et Flahault, filamento com falsa ramificação dupla mostrando heterocito (ponta de seta). 13. Symplocastrum friesii (C. Agardh) Forti. 14. Tolypothrix tenuis Kützing, filamento com falsa ramificação simples mostrando heterocito (ponta de seta). 15. Ecballocystis pulvinata Bohlin var. pulvinata, colônia dendróide mostrando camada gelatinizada da geração anterior (ponta de seta). 16. Spirogyra sp. 17. Zygnema sp. 18. Zygogonium ericetorum Kützing, tubo de conjugação. 19 e 21. Hydrosera whampöensis (Schwartz) Deby var. whampöensis. 19. Colônia formando filamento. 20. Vaucheria geminata (Vaucher) De Candolle in Lamarck et De Candolle, gametóforo mostrando oogônios (ponta de seta) e anterídeo (seta); 22-23. Batrachospermum ambiguum Montagne. 21. Vista valvar. 22. Ramo primário. 23. Carpogônio. 24. Estágio 'Chantransia' de Batrachospermum spp., filamento ramificado mostrando monosporângio (ponta de seta). Barras =19-20 = 50 $\mu \mathrm{m} ; 13,16-18,21$ e $24=20 \mu \mathrm{m} ; 12,14-15,22-23=10 \mu \mathrm{m}$. 
Material examinado: UPCB 53138, 53139, 53125, 53129, 53130, 53132.

Comentário: a grande variação métrica encontrada entre as diferentes populações na região de estudo sugere que as mesmas possam ser esporófitos de diferentes espécies de Batrachospermum spp. Porém, não há estudos conclusivos capazes de relacionar as características morfológicas do estágio "Chantransia" com o gametófito. Assim, não foi possível determinar a qual (ou a quantas) espécie pertence o material examinado.

Distribuição na Serra da Prata: Pontos de amostragem 1, 5, 7, 8 e 9.

Características dos riachos $(\mathrm{N}=4)$ temperatura da água $20,8-23,0{ }^{\circ} \mathrm{C}(\overline{\mathrm{x}}=21,8)$; condutividade $28-41 \mu \mathrm{S} . \mathrm{cm}^{-1}(\bar{x}=33) ; \mathrm{pH} 6,3-6,9$ $(\bar{x}=6,7)$; oxigênio dissolvido 5,1-6,0 mg.. $\mathrm{L}^{-1}$ $(\bar{x}=5,6)$; turbidez 1-3 NTU ( $\bar{x}=1,7)$; velocidade da correnteza 30-116 cm.s $\mathrm{c}^{-1} \quad(\bar{x}=84,8)$; profundidade $25,9-38,2 \mathrm{~cm}(\bar{x}=30,1)$; substrato rochoso; ambientes fortemente sombreados.

O levantamento taxonômico das espécies de macroalgas da Serra da Prata resultou, então, na identificação de 19 táxons (15 subgenéricos, três genéricos e uma fase do ciclo de vida de Batrachospermum spp.), os quais estão distribuídos em quatro divisões. Entre as divisões, Cyanophyta foi a mais representativa com $58 \%$ dos táxons encontrados, seguida pelas divisões Chlorophyta com $21 \%$, e por último Rhodophyta e Heterokontophyta com 10,5\% cada uma. Os táxons mais amplamente distribuídos na Serra da Prata foram Microcoleus subtorulosus (que ocorreu em nove amostras - 64\%), Phormidium aerugineo-caeruleum (oito amostras - 57\%) e o estágio 'Chantransia' de Batrachospermum spp. (seis amostras - 43\%).

Todas as espécies encontradas na Serra da Prata já foram descritas pela ciência e citadas para ambientes lóticos brasileiros. Porém, entre as espécies registradas, 53\% (10 espécies) tratam-se de novos registros para o Estado do Paraná, quais sejam: Blennothrix komarekii, Nostoc verrucosum, Nostochopsis lobatus, Pleurocapsa fluviatilis, Scytonema arcangeli, Tolypothrix tenuis, Ecballocystis pulvinata var. pulvinata, Zygogonium ericetorum, Vaucheria geminata e Batrachospermum ambiguum. Destas, Blennothrix komarekii, Nostoc verrucosum e Tolypothrix tenuis podem ser consideradas de ocorrência rara em ambientes lóticos brasileiros.

\section{Discussão}

O número total de táxons encontrados no presente estudo (19) pode ser considerado baixo quando comparado com aqueles de outros trabalhos utilizandose metodologias semelhantes: 31 táxons na região noroeste do Estado de São Paulo por Necchi Júnior et al. (1994), 42 na Mata Atlântica do Estado de São Paulo por Branco \& Necchi Júnior (1996a), 37 em três bacias de drenagem na região noroeste do Estado de São Paulo por Branco \& Necchi Júnior (1998) e 30 na Serra da Canastra em Minas Gerais por Necchi Júnior et al. (2003).

O táxon mais amplamente distribuído, Microcoleus subtorulosus, é recorrentemente mencionado em outros estudos (Branco \& Necchi Júnior 1996a; Necchi Júnior et al. 1997; Branco et al. 1999), porém é a primeira vez que tal espécie é mencionada como mais bem distribuída em uma região geográfica ampla. Por outro lado, Phormidium retzii que aparece em vários trabalhos como sendo a mais bem distribuída das espécies (e.g. Sheath \& Cole 1992; Necchi Júnior et al. 2000), na área de estudo só ocorreu em dois pontos de amostragem. Dos 19 táxons encontrados na Serra da Prata, 11 (57\%) foram encontrados uma única vez durante as amostragens, sugerindo uma tendência de ocorrência restrita das espécies a um ou poucos segmento do riacho. Tal tendência foi anteriormente observada em estudo desenvolvido no hemisfério norte (Hu \& Xie 2006).

A maior proporção de Cyanophyta em relação às demais divisões tem sido observada em alguns estudos sobre distribuição ecológica de macroalgas em ecossistemas lóticos (Filkin et al. 2003), porém, discorda com a grande maioria dos trabalhos que apontam Chlorophyta como a divisão predominante (Sheath \& Cole 1992; Necchi Júnior et al. 1995; Necchi Júnior et al. 2000; Hu \& Xie 2006). Ainda, a predominância de Cyanophyta no presente estudo está em aparente desacordo com os resultados de Branco \& Necchi Júnior (1996a) que encontraram Rhodophyta como grupo predominante em um estudo realizado no mesmo bioma no Estado de São Paulo. Os autores sugeriram que este resultado poderia ser explicado em função da atenuação da luz provocada pela presença de uma vegetação marginal exuberante, o que, em última análise, levaria ao melhor desempenho ecofisiológico do complexo pigmentar de tais algas. Entretanto, as algas azuis, que apresentam um complexo pigmentar muito semelhante ao das algas 
vermelhas, também podem ser consideradas como tendo um bom desempenho ecofisiológico em ambientes com limitação de luz (Sheath \& Burkholder 1985; Branco \& Necchi Júnior 1998). Assim, a similaridade nos pigmentos fotossintetizantes e no desempenho ecofisilógico em condições de baixa intensidade luminosa podem justificar a aparente divergência encontrada entre os resultados do presente estudo e o desenvolvido na Mata Atlântica paulista (Branco \& Necchi Júnior 1996a).

A comparação dos resultados obtidos no presente estudo com aqueles reportados para a Flona de Irati, uma unidade de conservação com predomínio de Floresta de Araucária no Estado do Paraná (Branco et al. Dados não publicados), evidencia um número relativamente baixo de táxons comuns às duas regiões (apenas nove táxons). Em adição, pode-se notar que as comunidades de macroalgas da Serra da Prata possuem uma diversidade de espécies menor do que aquela observada para a Flona de Irati, além do predomínio de algas azuis ao invés de algas verdes. Estes resultados sugerem que a composição das comunidades de macroalgas varia significativamente de uma região geográfica e/ou bioma para outra(o), mesmo quando as distâncias entre eles é relativamente pequena.

A grande proporção de novas ocorrências para o Estado (53\%), somada ao registro de algumas espécies raras, além da evidente distinção na composição florística observada nos ambientes lóticos da Serra da Prata, evidencia claramente a importância deste tipo de estudo para o conhecimento da biodiversidade e da biogeografia das comunidades de macroalgas de riachos, bem como para subsidiar trabalhos ecológicos e revisões taxonômicas com maior precisão.

\section{Agradecimentos}

Os autores agradecem aos funcionários do Parque Nacional Saint-Hilaire/Lange, pelo apoio durante os trabalhos de campo e pela disponibilidade da área de estudo; à FAPESP (proc. 09996-2), pelo apoio financeiro ao projeto; à CAPES, pela bolsa de mestrado a C.K. Peres; a Luis H. Zanini Branco, Orlando Necchi Júnior e Izabel C.A. Dias, pelas confirmações de parte das identificações.

\section{Referências bibliográficas}

Anagnostidis, K. \& Komárek, J. 1988. Modern approach to the classification system of cyanophytes. 3 . Oscillatoriales. Algological Studies 50-53: 327-472.
Anagnostidis, K. \& Komárek, J. 1990. Modern approach to the classification system of cyanophytes. 3 . Stigonematales. Algological Studies 59: 1-73.

Azevedo, M.T.P.; Nogueira, N.M.C. \& Sant'anna, C.L. 1996. Criptógamos do Parque Estadual das Fontes do Ipiranga, São Paulo, SP. Algas, 8: Cyanophyceae. Hoehnea 23: 1-38.

Branco, C.C.Z.; Branco, L.H.Z.; Moura, M.O. \& Bertusso, F.R. 2005. The sucession dynamics of a macroalgal community after a flood disturbance in a tropical stream from São Paulo State, Southeastern Brasil. Revista Brasileira de Botânica 28: 267-275.

Branco, C.C.Z. \& Necchi Júnior, O. 1996a. Survey of stream macroalgae of eastern Atlantic Rainforest of São Paulo State, southeastern Brazil. Algological Studies 80: 35-57.

Branco, C.C.Z. \& Necchi Júnior, O. 1996b. Distribution of stream macroalgae in the eastern Atlantic Rainforest of São Paulo State, southeastern Brazil. Algological Studies 333: 139-150.

Branco, C.C.Z.; Necchi Júnior, O. \& Branco, L.H.Z. 2002. Taxonomy and ecological distribution of Chaetophoraceae (Chaetophorales, Chlorophyta) in lotic ecosystems from São Paulo State, southeastern Brazil. Algological Studies 106: 43-75.

Branco, L.H.Z. \& Montejano, G. 2006. A new morphotype of Blennothrix (Cyanoprokariota, Oscillatoriales) from streams of Brazil and Mexico. Algolgical Studies 121: 35-42.

Branco, L.H.Z. \& Necchi Júnior, O. 1998. Distribution of macroalgae in three tropical drainage basins of southeastern Brazil. Archiv für Hydrobiologie 142: 241-256.

Branco, L.H.Z.; Necchi Júnior, O. \& Branco, C.C.Z. 1999. Cyanophyta from lotic ecosystems of São Paulo State, southeastern Brazil. Algological Studies 94: 63-87.

Branco, L.H.Z.; Necchi Júnior, O. \& Branco, C.C.Z. 2001. Ecological distribution of Cyanophyceae in lotic ecossystems of São Paulo State. Revista Brasileira de Botânica 24: 99-108.

Brito, E.F.; Moulton, T.P.; Souza, M.L. \& Bunn, S.E. 2006. Stable isotope analysis indicates microalgae as the predominant food source of fauna in a coastal Forest stream, south-east Brazil. Austral Ecology 31: 623-633.

DeNicola, D.M.; Hogland, K.D. \& Roemer, S.C. 1992. Influence of canopy cover on spectral irradiance and periphyton assemblages in a prairie stream. Journal of the North American Benthological Society 11: 391-404.

Dias, I.C.A. 1986. Zygnemaceae (Zygnemaphyceae) da Chapada dos Guimarães e arredores, Mato Grosso, Brasil: uma contribuição ao seu conhecimento. Rickia 13: 69-75.

Dias, I.C.A. 1987. Algas do Bosque Arruda Câmara, Rio de Janeiro, Brasil: Chlorophyta filamentosas. Rickia 14: 45-51.

Dias, I.C.A. 1990. Sobre algumas Chlorophyta filamentosas da Fazenda Água Limpa, Distrito Federal, Brasil. Hoehnea 17: 51-61.

Dias, I.C.A. 1991. Estudos ficológicos na Região Noroeste Brasileira: Chlorophyta filamentosas. Hoehnea 18: 157-169. 
Dias, I.C.A. 1992. Algas continentais do Estado do Rio de Janeiro, Brasil: Oedogoniaceae e Zygnemaceae. Hoehnea 19: 51-63.

Filkin, N.R.; Sherwood, A.R. \& Vis, M.L. 2003. Macroalgae from 23 Stream in the Hawaiian Islands. Pacific Science 57: 421-431.

Giller, P.S. \& Malmqvist, B. 1998. The Biology of Streams and Rivers: biology of habitat. Oxford, Oxford University Press.

Gordon, N.D.; McMahon, T.A. \& Finlayson, B.L. 1992. Stream Hydrology: an introduction for ecologists. Chichester, John Wiley \& Sons.

Hoek, C. Van den; Mann, D.G. \& Jahns, H.M. 1995. Algae: an introduction to phycology. Cambridge, Cambrige University Press.

Hu, B.F. \& Xie, S.L. 2006. Effect of seasonality on distribution of macroalgae in a stream system (Xin'an Spring) in Shanxi Province, North China. Journal of Integrative Plant Biology 48: 889-896.

IBAMA. 2003. Instituto Brasileiro do Meio Ambiente e Recursos Naturais Renováveis. Apresentação. In: Guia Philips - Parques Nacionais. $2^{\mathrm{a}}$ ed. São Paulo, Horizonte Geográfico.

IBGE. 1992. Instituto Brasileiro de Geografia e Estatística. Manual Técnico da Vegetação Brasileira. Série Manuais Técnicos em Geociências, Rio de Janeiro, n. 7.

Komárek, J. \& Anagnostidis, K. 1986. Modern approach to the classification system of cyanophytes. 2. Chroococcales. Algological Studies 43: 157-226.

Komárek, J. \& Anagnostidis, K. 1989. Modern approach to the classification system of cyanophytes. 4. Nostocales. Algological Studies 56: 247-345.

Komárek, J. \& Anagnostidis, K. 2005. Cyanoprokaryota, part 2. Oscillatoriales. Süsswasserflora von Mitteleuropa Band 19/2 (B. Büdel; G. Gärtner; L. Krienitz \& M. Schagerl, eds.). Jena, Gustav Fischer.

Matthael, C.D.; Guggelberger, C. \& Huber, H. 2003. Local disturbance history affects patchiness of benthic river algae. Freshwater Biology 48: 1514-1526.

Necchi Júnior, O. 1989. Rhodophyta de água doce do Estado de São Paulo: levantamento taxonômico. Boletim de Botânica da Universidade de São Paulo 11: 11-69.

Necchi Júnior, O. \& Bicudo, D.C. 1992. Criptógamos do Parque Estadual das Fontes do Ipiranga, São Paulo, SP. Algas, 3: Rhodophyceae. Hoehnea 19: 89-92.

Necchi Júnior, O.; Branco, C.C.Z. \& Branco, L.H.Z. 2000. Distribution of stream macroalgae in São Paulo State, southeastern Brazil. Algological Studies 97: 43-57.
Necchi Júnior, O.; Branco, C.C.Z.; Simões, R.C.G. \& Branco, L.H.Z. 1995. Distribution of stream macroalgae in northwest region of São Paulo State, southeastern Brazil. Hydrobiologia 299: 219-230.

Necchi Júnior, O.; Branco, L.H.Z. \& Branco, C.C.Z. 2003. Ecological distribution of stream macroalgal communities from a drainage basin in the Serra da Canastra National Park, Minas Gerais, Southeastern Brazil. Brazilian Journal of Biology 63: 1-12.

Necchi Júnior, O.; Dip, M.R. \& Góes, R.M. 1991. Macroalgae of a stream in southeastern Brazil: composition, seasonal variation and relation to physical and chemical variables. Hydrobiologia 213: 241-250.

Necchi Júnior, O.; Pascoaloto, D.; Branco, C.C.Z. \& Branco, L.H.Z. 1997. Stream macroalgal flora from the northwest region of São Paulo State, southeastern Brasil. Algological Studies 84: 91-112.

Necchi Júnior, O.; Pascoaloto, D. \& Branco, L.H.Z. 1994. Distribution of macroalgae in a tropical river basin from southeastern Brazil. Archiv für Hydrobiologie 129: 459-471.

Pró-Atlântica. 2002. Zoneamento Ecológico-Econômico e Plano de Gestão da APA de Guaratuba. Relatório de consultoria apresentado por Harald Müeller, Curitiba.

Roderjan, C.V.; Galvão, F.; Kuniyoshi, Y.S. \& Hatschbach, G.G. 2002. As unidades fitogeográficas do Estado do Paraná. Ciência \& Ambiente 24: 75-92.

Sant'Anna, C.L. 1988. Scytonemataceae (Cyanophyceae) from the State of São Paulo, southern Brazil. Nova Hedwigia 46: 519-539.

Sheath, R.G. \& Burkholder, J. 1985. Characteristics of softwater stream in Rhode Island. II: Composition and seasonal dynamics of macroalgae communities. Hydrobiologia 128: 109-118.

Sheath, R.G. \& Cole, K.M. 1992. Biogeography of stream macroalgae in North America. Journal of Phycology 28: 448-460.

Sheath, R.G.; Hambrook, J.A. \& Nerone, C.A. 1988. The benthic macro-algae of Georgia Bay, the North Channel and their dranaige basin. Hydrobiologia 163: 141-148.

Sheath, R.G.; Hamilton, P.B.; Hambrook, J.A. \& Cole, K.M. 1989. Stream macroalgae of eastern boreal forest region of North America. Canadian Journal of Botany 67: 3553-3562.

Sheath, R.G.; Morison, M.O.; Korch, J.E.; Kaczmarczyk, D. \& Cole, K.M. 1986. Distribution of stream macroalgae in south-central Alaska. Hydrobiologia 135: 259-269.

Sherwood, A.R. \& Sheath, R.G. 1999. Seasonality of macroalgae and epilithic diatoms in spring-fed streams in Texas, U.S.A. Hydrobiologia 390: 73-82. 